\title{
MR IMAGING IN CHRONIC HEPATIC ENCEPHALOPATHY
}

\author{
Vasant Devkar ${ }^{1}$ Paritosh Desai ${ }^{2}$
}

${ }^{1}$ Associate Professor, Department of Medicine, Krishna Institute of Medical College and Hospital. ${ }^{2}$ Resident, Department of Medicine, Krishna Institute of Medical College and Hospital.

\section{ABSTRACT}

Hepatic encephalopathy reflects a spectrum of neuropsychiatric abnormalities occurring in the patients with liver dysfunction. ${ }^{1}$ Here, we discuss a 60-year-old male in hepatic encephalopathy with features of chronic hepatic encephalopathy on MR imaging.

\section{KEYWORDS}

Encephalopathy, MR Imaging, Chronic Hepatic, Encephalopathy.

HOW TO CITE THIS ARTICLE: Devkar V, Desai P. MR imaging in chronic hepatic encephalopathy. J. Evolution Med. Dent. Sci. 2016;5(79):5924-5926, DOI: 10.14260/jemds/2016/1337

\section{INTRODUCTION}

Hepatic encephalopathy manifests as a neuropsychiatric syndrome encompassing a wide spectrum of mental and motor disorder, the changes in mental status range from subtle cognitive dysfunction to severe coma, whereas the motor function changes include rigidity, disorder of speech production, resting and movement induced tremors. Hepatic encephalopathy may result from hepatic insufficiency from acute liver failure or cirrhosis or from portosystemic shunting, sometimes even in the absence of intrinsic liver disease. ${ }^{2}$ Pathogenesis of this condition is not well defined. ${ }^{3}$ The aim of this case report was to present a case of hepatic encephalopathy and discuss in detail the features of chronic hepatic encephalopathy on MR imaging and its treatment.

\section{CASE REPORT}

A 60-year-old male patient was brought to emergency room with chief complaints of altered sensorium, irrelevant talk and generalised weakness since 2-3 days. History of similar complaints in the past, 5 years back (did not receive any treatment for the same, recovered spontaneously), known case of hypertension not on any treatment, chronic alcoholic since last 15 years, last bout 5 months back.

On general examination at the time of admission the patient was lethargic and confused, disoriented to time, place and person. Vitally patient was stable, HR-108/min, BP130/90 mmHg, RR-20/min, afebrile. Pallor was present, icterus was present, no peripheral oedema.

\section{On Systemic Examination}

Cardiac and respiratory status was normal. P/A - soft, distended, free fluid present, shifting dullness present, no organomegaly, no dilated veins. CNS - conscious, disoriented to time, place and person. Power - moving all 4 limbs, tone normal, reflexes normal, plantar left extensor.

\section{INVESTIGATIONS}

Haemogram was suggestive of mild iron deficiency anaemia,

Financial or Other, Competing Interest: None.

Submission 25-08-2016, Peer Review 19-09-2016,

Acceptance 24-09-2016, Published 03-10-2016.

Corresponding Author:

Dr. Vasant Devkar,

496A, Shaniwarpeth,

Karad-415110.

E-mail: vasantvd@gmail.com

DOI: $10.14260 /$ jemds $/ 2016 / 1337$

\section{(c) (i) $\$$}

PT - 28.5, APTT- 36, INR - 2.8, BT 2.6, CT 6.6, RFT are WNL, LFT was WNL (bil total-0.9, bil direct-0.5, SGOT 51, SGPT 46), serum proteins was on a slight lower side (protein total-5.6, albumin-3), serum ammonia- 90.3 (normal 30-127), ESR - 25. CXR - WNL, ECG - WNL, USG A/P - Liver appears normal in size shows surface nodularities with altered parenchymal echotexture (cirrhotic changes), no local lesions, IHBR not dilated, few periportal collaterals noted. Spleen is mildly enlarged few perisplenic collaterals noted. Impressioncirrhotic changes in liver with features of portal hypertension.

\section{MRI Brain (Plain)}

Symmetrical areas of altered signal intensity in bilateral globi pallidi, posterior limb of internal capsule, subthalamic nuclei and cerebral peduncle, hyperintense on T1/FLAIR, isohypointense on $\mathrm{T} 2 \mathrm{~W}$ showing no restriction of diffusion. Multiple focal areas of hyperintensity on T2W1/FLAIR are seen in the periventricular white matter, subcortical white matter, centrum semiovale and corona radiata which are hypointense on T1W1 not showing restriction of diffusion most likely suggestive of old white-matter ischaemic changes. Brain stem shows normal morphology and signal intensity. No intra-axial collection.

Patient was diagnosed early and hence responded well to the conservative treatment and patient's mental status continued to improve over a period of 4 days and shifted towards for further management as he did not need ICU care and on the 9th day of admission patient was discharged from the wards.

\section{DISCUSSION}

Chronic hepatic encephalopathy classically present with small lesions in specific areas of the brain, mainly the white matter and basal ganglia and pons. These are areas supplied by small perforating blood vessels and are often associated with hypertensive disease or other angiopathies. ${ }^{4}$ In hepatic encephalopathy, these small cerebral blood vessels may be preferentially affected. Susceptibility changes were especially prominent in the corpus callosum, which may be due to the fact that the callosal white matter fibres are closely packed together, so that the penetrating vessels have even smaller diameters and less compliance, therefore may be at increased risk of developing intravascular thrombi or microhaemorrhages. There is also alteration in cerebral blood flow, which may also be responsible in part for brain hyperaemia resulting in cerebral blood flow. 
The lack of autoregulation of CBF in liver failure patients is believed to be due to hyperammonia in combination with dilation of cerebral arterioles and cerebral vasoparalysis. ${ }^{5}$ These changes in microvasculature could also be the reason that small arterioles are more susceptible to injury and create the hypodensities, which can be detected by SW1. ${ }^{6}$ Cerebral blood flow and brain oedema are most probably the aetiology of the lesions found in this case, but presence of hepatic coagulopathy also has to be ruled out. ${ }^{7}$

\section{Management}

To exclude other causes of altered sensorium. -Providing supportive care. - Identifying and treating precipitating causes. -Reduce nitrogenous load in the gut. - Assessing the need for long-term therapy and liver transplant evaluation. Prevention of recurrent hepatic encephalopathy. -Medical therapy includes proper hydration, correction of electrolytes, non-absorbable disaccharides such as lactulose, antibiotic (rifaximin) is a reasonable alternative in those who do not respond to lactulose alone. Nutrition includes dietary protein restriction.

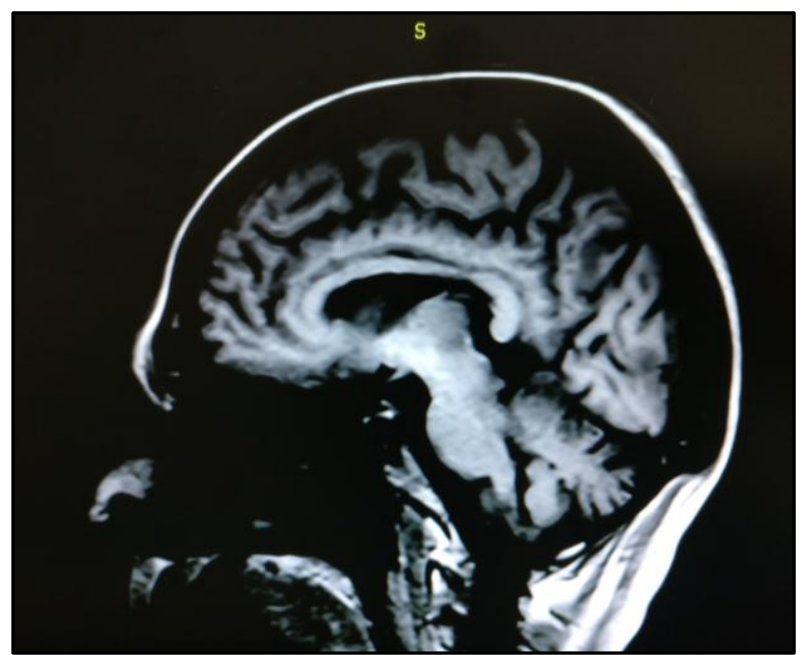

Fig. 1: Altered Signal Intensities in Globi Pallidi, Posterior Limb of Internal Capsule, Subthalamic Nuclei and Cerebral Peduncle

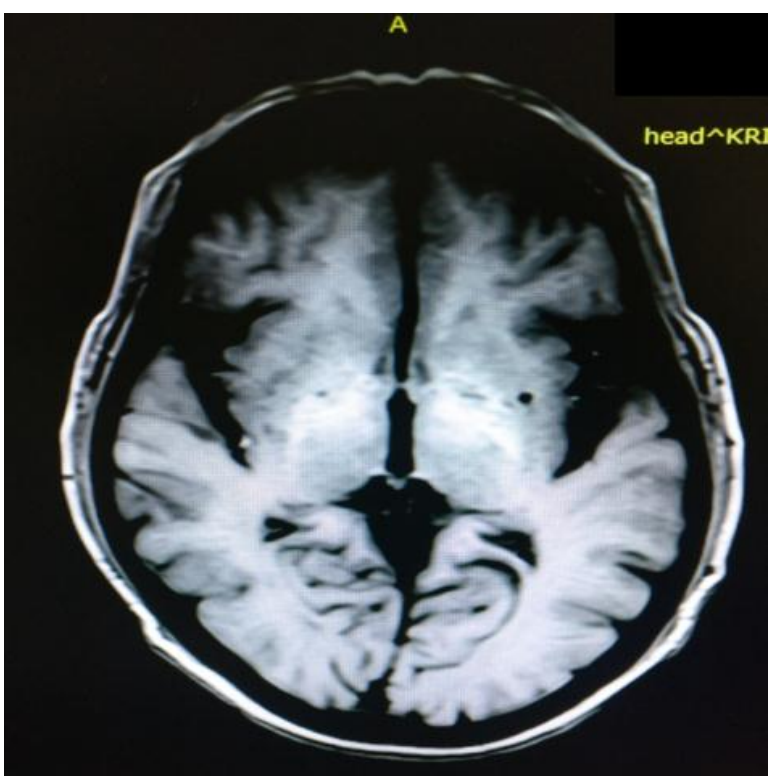

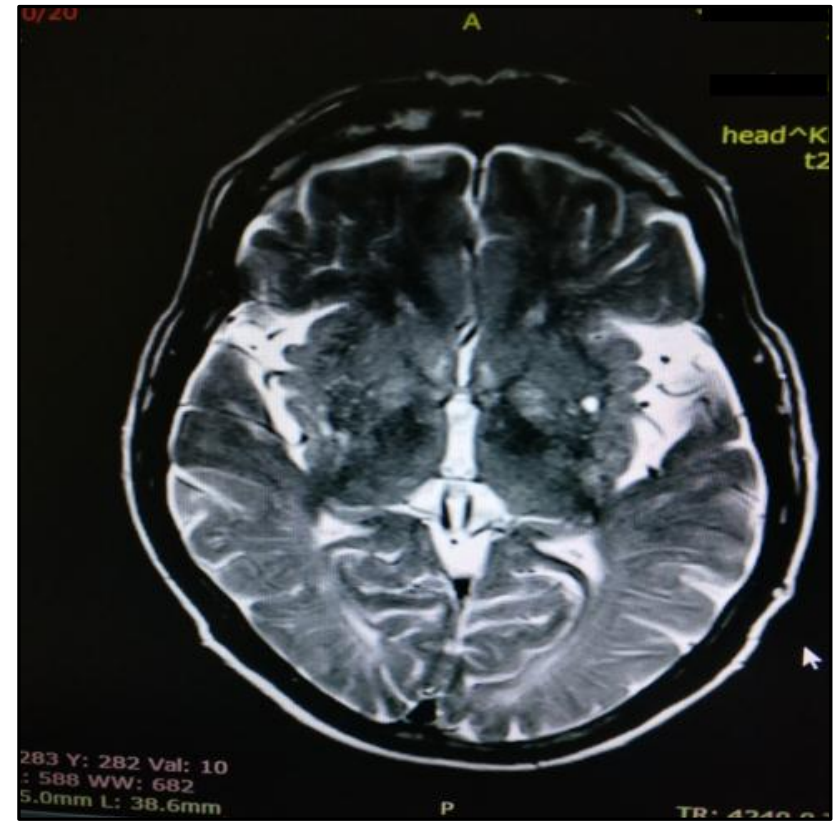

Fig. 2 \& 3: Hyperintense Posterior Limb of Internal Capsule on T1/Flair

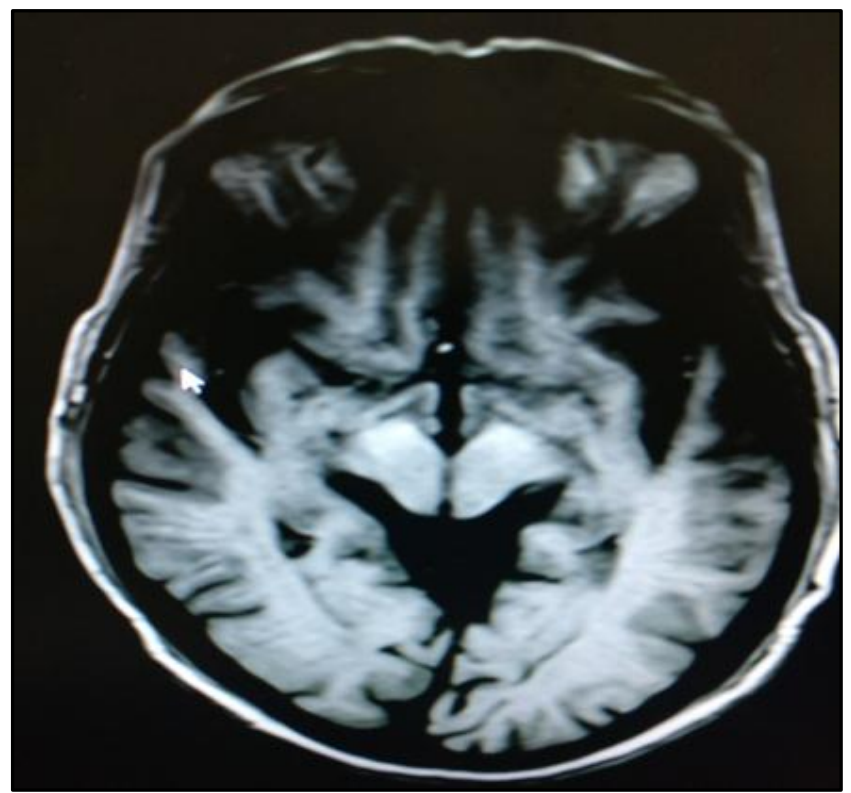

Fig. 4: Hyperintense Signals in Bilateral Subthalamic Nuclei

\section{REFERENCES}

1. American Association for the Study of Liver Diseases: European Association for the Study of the Liver. Hepatic encephalopathy in chronic liver disease: 2014 practice guideline by the European Association for the Study of the Liver and the American Association for the Study of Liver Diseases. J Hepatol 2014;61(3):642-59.

2. Rovira A, Cordoba J, Raguer N, et al. magnetic resonance imaging measurement of brain edema in patients with liver disease: resolution after transplantataion. Current Opin Neurol 2002;15(6):731-7.

3. Rovira A, Alonso J, Cordoba J. MR imaging findings in hepatic encephalopathy. AJNR Neuroradiol 2008;29(9):1612-21. 
4. Wijdicks EF, Plevak DJ, Rakela J, et al. Clinical and radiological features of cerebral edema in fulminant hepatic failure. Mayo Clinic Proc 1995;70(2):119-24.

5. Miranda M, Caballero L. Chronic hepatic encephalopathy: the role of high serum manganese levels and its relation with basal ganglia lesions in nuclear magnetic resonance of the brain. Clinical case. Rev Med Chil 2001;129(9):1051-5.
6. Ahl B, Weisseborn K, van den Hoff J, Fischer-Wasleys D, et al. Regional differences in cerebral blood flow and the cerebral ammonia metabolism in the patients with cirrhosis. Hepatology 2004;40(1):73-9.

7. Larsen FS. Cerebral blood flow in hyperammonemia: heterogenecity and starling forces in capillaries. Metab Brain Dis 2002;17(4):229-35. 